\title{
The Versatile Approach: A Novel Single Incision Combined with Anterior and Posterior Approaches for Decompression and Instrumented Fusion to Treat Tuberculosis of the Thoracic Spine
}

\author{
Sudhir Kumar Srivastava, Rishi Anil Aggarwal, Sunil Krishna Bhosale, Kunal Roy, Pradip Sharad Nemade
}

Department of Orthopaedics, Seth G.S. Medical College and KEM Hospital, Maharashtra, India

\begin{abstract}
Study Design: Retrospective case series.
Purpose: To describe a novel single incision that combines anterior and posterior approaches for decompression and instrumented fusion to treat tuberculosis of the thoracic spine and study the neurological and radiological outcomes.

Overview of Literature: Tuberculosis of the spine remains a major health issue in many developing countries. The options for treating tuberculosis of the thoracic spine include the anterior, posterior, and combined approaches, each with its advantages and disadvantages.

Methods: Totally, 143 patients with tuberculosis of the thoracic spine were surgically treated using the "Versatile approach". Posterior fixation was performed using sublaminar wires and a Hartshill rectangle in all patients. Anterior reconstruction was accomplished using bone graft harvested from autologous rib, iliac crest, or fibula.

Results: The study included 45 males and 98 females, with a mean age of 33.18ะ18.65 years (range, 3-82 years) and a mean followup of $60.23 \pm 24.56$ months (range, 18-156 months). Kyphosis improved from a mean value of 24.02 preoperatively to 10.25 postoperatively. A preoperative neurological deficit was observed in 131 patients, with 130 patients regaining ambulatory power. No patient had deterioration of neurological status following surgery. Fusion was achieved in all cases. The visual analogscale score improved from an average score of 7.02 preoperatively to 1.51 at final follow-up. Eight patients had superficial macerations, which healed spontaneously. One patient had buckling of the anterior graft, and one patient had implant breakage following road traffic accident. Conclusions: The "Versatile approach" is an effective, single-stage, single-incision method that combines anterior and posterior approaches for the surgically treating tuberculosis of the thoracic spine. It offers the advantage of direct visualization for decompression and reconstruction of the anterior and posterior vertebral columns, thus providing an excellent, long-lasting clinical outcome.
\end{abstract}

Keywords: Thoracic spine; Tuberculosis; Single incision; Combined approach; Fusion

\section{Introduction}

Tuberculosis is a major health issue in many developing countries. Although pulmonary tuberculosis is the most common form of tuberculosis, extrapulmonary tuberculosis accounts for $15 \%-20 \%$ of all tuberculosis cases

\footnotetext{
Received May 14, 2016; Revised Oct 12, 2016; Accepted Nov 1, 2016

Corresponding author: Rishi Anil Aggarwal

Department of Orthopaedics, Seth G.S. Medical College and KEM Hospital, Parel, Mumbai-400012, Maharashtra, India

Tel: +91-22-410-7463, Fax: +91-22-410-7463, E-mail: ris1987@gmail.com
} 
[1]. Bone and joint tuberculosis compose the third-most common form of extrapulmonary tuberculosis [2]. Tuberculosis of the spine is the most common form of skeletal tuberculosis [3]. The most commonly affected site is the dorsolumbar spine, with $20 \%-41 \%$ of spinal tuberculosis cases developing neurological deficits [4]. Tuberculosis of the dorsal spine is most commonly associated with neurological deficits due to the narrower spinal canal and physiological kyphosis, which forces necrotic tissue into the spinal canal.

Most patients with tuberculosis of the dorsal spine are treated with ambulatory chemotherapy. Neurological deficits, unstable vertebral column, and conservative management failure are indications for surgical treatment, which include anterior, posterior, and combined approaches [59]. We describe the "Versatile approach", a novel surgical approach, which is a single incision that combines anterior and posterior approaches for decompression and instrumented circumferential fusion to treat tuberculosis of the thoracic spine.

We have used this approach extensively in patients with tuberculosis of the dorsal spine. We believe that good anterior reconstruction is extremely important for successful treatment. The direct anterior approach via thoracotomy allows visualization of anterior column for excellent debridement and reconstruction. However, fixation by this approach is biomechanically inferior to that by posterior approach. The posterior approach allows for good fixation, but anterior debridement and reconstruction using this approach requires sacrificing a part of the normal posterior element to expose the anterior column. We used the "Versatile approach" for fixation of the tensile posterior surface and direct visualization of anterior debridement and reconstruction using a single incision and single stage without changing the patient position.

\section{Materials and Methods}

Between January 2000 and December 2013, 143 consecutive patients with tuberculosis of the dorsal spine underwent surgery. Operation theatre reports, patient records, and radiographs were retrospectively reviewed with permission from the Institutional Review Board. Indications for surgery were neurologic deficit presence (131 patients) and unstable vertebral column (12 patients). We defined an unstable vertebral column as complete loss of $>1$ vertebral body with localized kyphosis of $>30^{\circ}$, with or without coronal/sagittal plane translation (Fig. 1A). As per our institutional protocol, all patients with Frankel grade $\mathrm{C}$ or $\mathrm{D}$ were on bed rest and underwent chemotherapy for a minimum period of 3 and 6 weeks, respectively, before being considered for surgery. This minimum period of 3 weeks is considered the "middle path regime" popularized by Tuli [10]. Surgery is considered if patients fail to improve after 3-4-weeks anti-tuberculosis therapy (ATT) and bed rest [11]. Only patients who did not recover or deteriorated while on conservative treatment were considered for surgery. Patients with Frankel grade A or B underwent surgery as early as possible.

Patient age, gender, diagnosis, vertebral levels involved, levels fused, bone type used for anterior reconstruction, preoperative and postoperative kyphosis angles ( $\mathrm{K}$ angle), correction loss at follow-up, preoperative and postoperative neurological status (Frankel Grading), preoperative and postoperative pain assessed by the visual analog scale (VAS) score, estimated blood loss, and postoperative complications were noted.

\section{Radiological evaluation}

Preoperative radiographs included standing anteroposterior (AP) and lateral views of the dorsolumbar spine. In patients with no ambulatory power in their lower limbs and imminent paralysis, supine radiographs were ac-
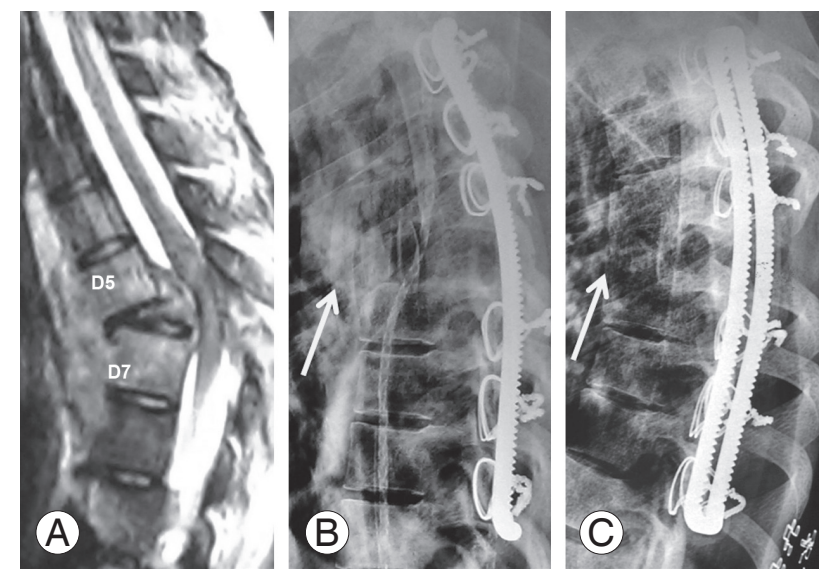

Fig. 1. Imaging of a patient during the "Versatile approach". (A) Preoperative image showing D5-D7 tuberculosis of the spine with D6 vertebra collapse and localized kyphosis of $40^{\circ}$. (B) Postoperative $X$-ray (lateral view) showing kyphosis correction. Anterior reconstruction was performed using a tricortical bone graft. Part of the excised rib was used as an onlay graft (arrow). (C) X-ray at 1 year follow-up, showing anterior fusion. The onlay graft has fused with the vertebral bodies (arrow). 
quired. Preoperative magnetic resonance imaging (MRI) was performed for all patients. AP and lateral radiographs of the dorsolumbar spine were performed postoperatively and during follow-up at 3, 6, 12, and 24 months. The $\mathrm{K}$ angle was calculated by drawing lines from the upper to the lower border of the first normal vertebra above and below the diseased area, respectively. The $\mathrm{K}$ angle does not represent overall thoracic kyphosis but it is a measure of the localized kyphosis due to the anterior column collapse from tuberculosis. Computed tomography was performed 1 year postoperatively in patients in whom fusion could not be assessed on X-ray. Anterior fusion was assessed by the presence of bone bridging the vertebrae immediately proximal and distal to the reconstructed area.

\section{Statistical analysis}

Descriptive statistics, including the means and standard deviations, were calculated for variables using SPSS software ver. 17.0 (SPSS Inc., Chicago, IL, USA). The preoperative $\mathrm{K}$ angle and VAS score were compared with postoperative values using a paired $t$ test.

\section{Surgical procedure}

\section{1) Positioning of the patient}

The thoracic spine can be approached from the left or right side. The upper thoracic spine is generally approached from the right side because of aortic arch location, but it can be approached from the left side without any difficulty if needed.

The patient is positioned on their left side, with their right arm placed as far toward the head as possible. This is important for pulling the scapula anteriorly and superiorly.

\section{2) Incision and anterior exposure}

A semilunar incision is made in the right paramedian region. The incision ends are gradually slanted and run close to the midline, extending along the entire length of the proposed area of fixation (Fig. 2). The semilunar curve apex lies just proximal to the diseased dorsal vertebral level and approximately $8-10 \mathrm{~cm}$ away from the midline.

In 2014, we presented a study at the Western Indian Regional Orthopaedic Conference, discussing MRI results from 100 patients. Majority patients has abscesses on the right side, which were thought to be due to less resistance by venous structures on the right side compared with that by the aorta on the left side. Because surgery is performed with the patient in the lateral position, it is easier for a right-handed surgeon to pass a sublaminar wire from the distal to the proximal edge of the lamina. However, if there is preoperative evidence of intrathoracic fibrosis on the right side, a left-sided approach may be preferred.

The skin, subcutaneous tissue, deep fascia, and trapezius are cut along the skin incision. If required, the latissimus dorsi muscle is divided as far caudally as possible. The rhomboids are incised toward the midline to avoid spinal accessory nerve and transverse cervical artery damage. If required, the serratus anterior muscle is divided as far caudally as possible to avoid long thoracic nerve damage. Stay sutures in the muscle help in proper approximation during closure. Here, the deep semilunar flap is mobilized toward the midline, exposing the spinous processes (Fig. 3). The scapula and attached muscle

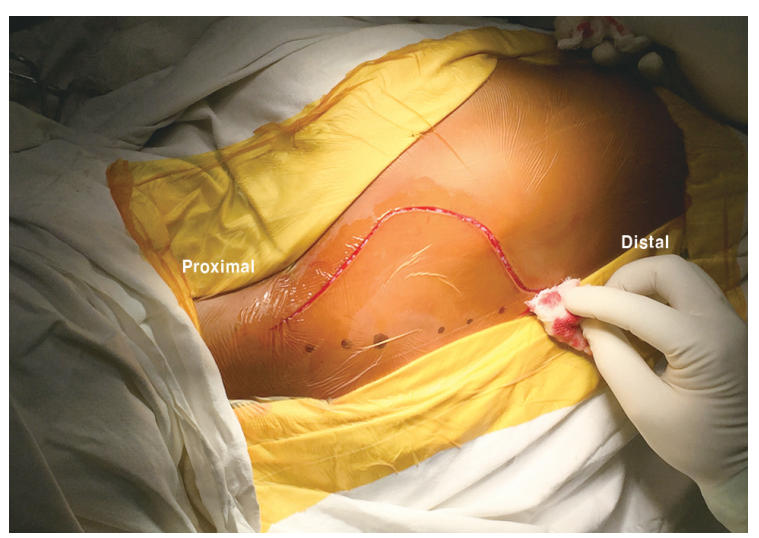

Fig. 2. The semilunar incision. The ends of the incision gradually slant and run close to the midline, extending along the length of the proposed area of fixation.

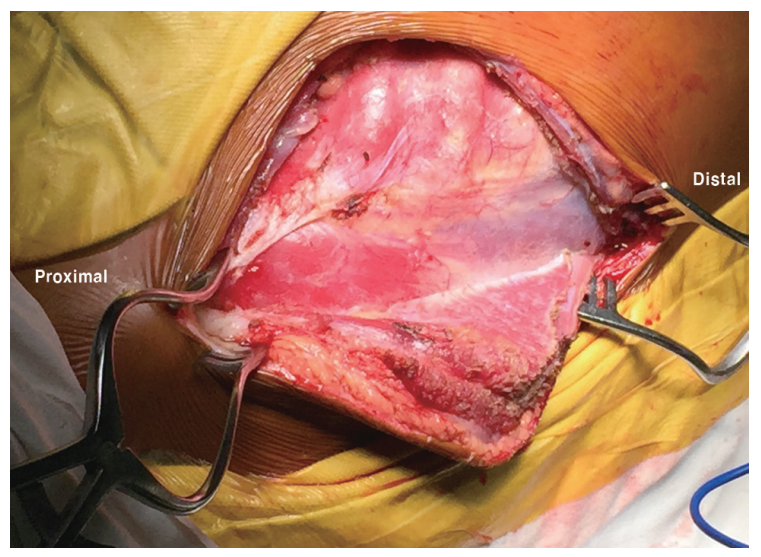

Fig. 3. The deep semilunar flap is mobilized toward the midline, exposing the spinous processes. 
are retracted anteriorly, exposing the rib cage outer surface for assessing the particular rib level. The paraspinal muscle is partially reflected, without severing, from the lateral to medial sides at the proposed rib resection level. The rib corresponding to the collapsed vertebral level is then excised. The rib periosteum is incised, and then a subperiosteal rib excision is performed by cutting the rib as anterior as possible and controlling the posterior end delivery by gently reflecting the paraspinal muscle, cutting the costotransverse ligament, and gently rocking the head of the rib from the vertebral body. This is quite easy in tuberculosis cases because there is existing damage in this region, and delivery of the posterior end usually leads to cold abscess drainage. At this stage, an extrapleural or transpleural approach is used to reach the anterior surface of the vertebral body (Fig. 4). A chest spreader is used to increase the space; the lung is retracted on a mop with a Deaver retractor. Segmental vessels are subsequently exposed and ligated. We routinely ligate the vessels (not just coagulate) to prevent a secondary hemorrhage because segmental vessels are very close to the aorta and the coagulated end might open if the blood pressure increases postoperatively.

After exposing the thoracic vertebra by the anterior approach, the "critical supply zone" of the spinal cord (the watershed zone) should be examined. The watershed zone

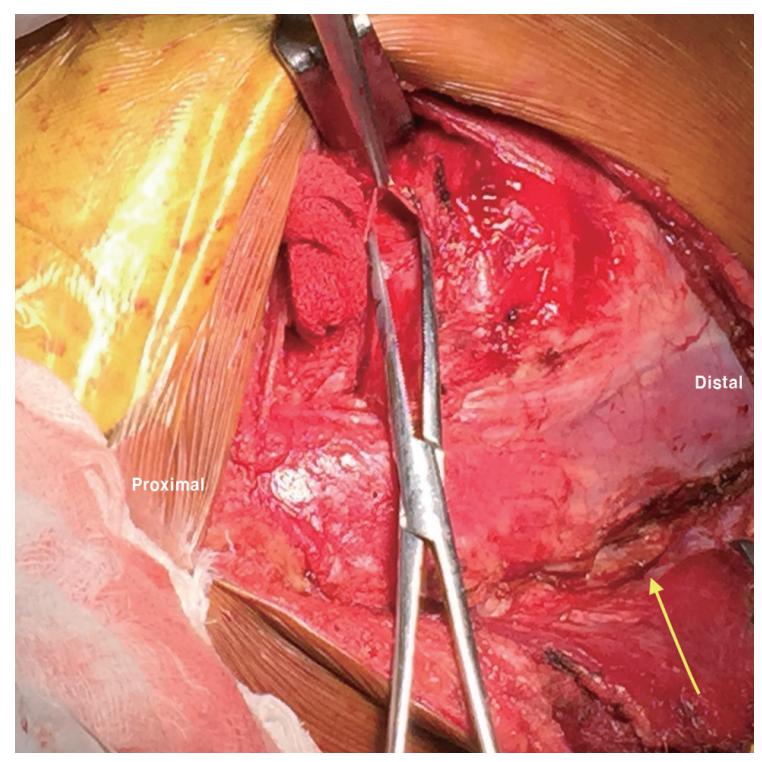

Fig. 4. An incision is made through the parietal pleura, and the opening is dilated using artery forceps prior to cutting the remaining pleura. The exposed part of a spinous process is seen toward the midline (yellow arrow). generally lies between the fourth and ninth thoracic vertebrae. The segmental arteries in these vertebrae should be transected as far anterior as possible. Segmental vessel ligation near the intervertebral foramen should be avoided to avoid damaging the blood supply to the spinal cord, particularly in the watershed area.

3) Decompression, posterior exposure, and instrumentation Debridement and debulking of the anterior necrotic tissue are then performed. Any fibrosis that bridges the right and left intervertebral spaces should be disrupted for ease in localized kyphosis correction using posterior instrumentation. Following the initial debridement, an interbody spreader is used to control the anterior column if it collapses (Fig. 5).

The lung is then inflated. Posterior exposure of the spinous process, laminae, transverse processes, and facet joints is performed by separating the erector spinae muscle on the left and right sides (Fig. 5). If the laminae are involved, a laminectomy may be performed.

At this stage, posterior instrumentation is used. The use of sublaminar wires and a Hartshill rectangle (rectangle loop) is convenient, provides good segmental fixation, and corrects the existing deformity (Fig. 6). If contralateral posterolateral decompression is required, it should be

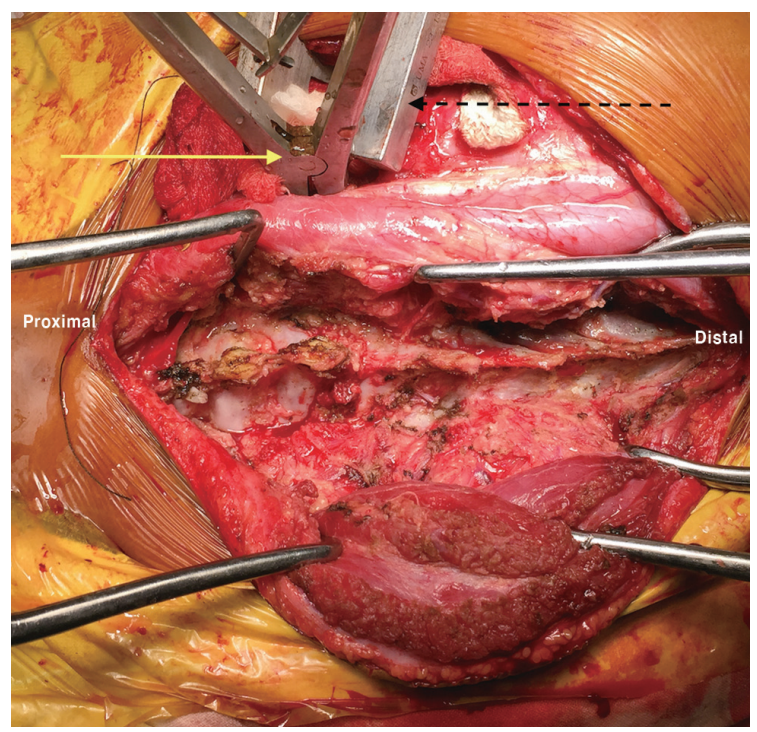

Fig. 5. Following the initial anterior lesion debridement, an interbody spreader is inserted between the proximal and distal vertebrae (yellow arrow, interbody spreader; dashed arrow, chest wall spreader). Posterior exposure is achieved. The dashed arrow shows the chest wall spreader used to open the chest cavity during the anterior procedure. 


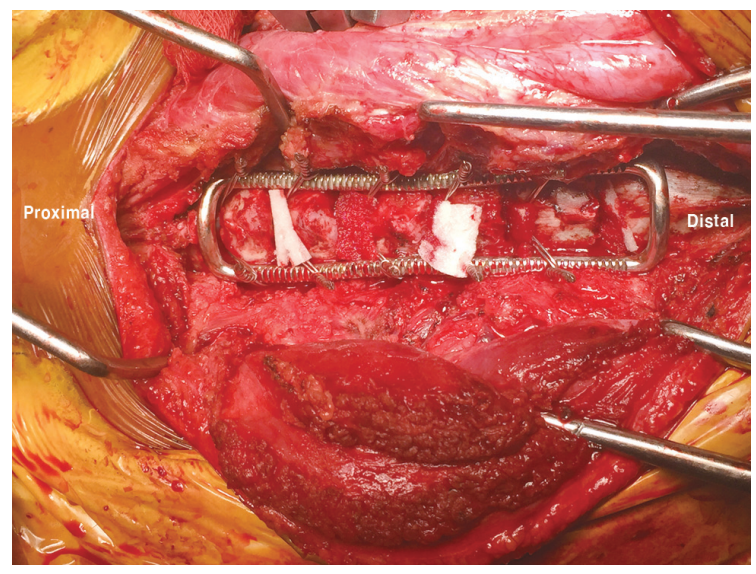

Fig. 6. Posterior instrumentation performed using sublaminar wires and a Hartshill rectangle.

performed before placing the Hartshill rectangle. One can also use a hook/rod system in this position.

One can then return to the anterior region after retracting the lung. Following the use of instrumentation, the anterior gap widens. Final debridement is then performed. The posterior longitudinal ligament (PLL) is excised in cases of dense neurological deficit and if obvious anterior compression is still present after necrotic tissue removal.

\section{4) Reconstruction}

The quality of the remnant bone is then assessed. If the remnant bone is $>5 \mathrm{~mm}$ and has not been breached by the tuberculous abscess, then it is salvaged. If the remnant bone is $<5 \mathrm{~mm}$ or has been breached by the tuberculous abscess, then it is excised, along with the adjoining disk, until the adjacent vertebral endplate can be seen. A cutoff of $5 \mathrm{~mm}$ is chosen because if the remnant bone is $<5$ $\mathrm{mm}$ or has been breached by necrotic tissue, the chances of a nonunion are increased; in such a scenario, it is better to excise such a remnant and perform the reconstruction using healthy bone. A proper slot is made in the proximal and distal regions of the healthy bone; strut grafting is performed. Anterior to the strut graft, small corticocancellous chips are packed. An additional onlay graft (part of the excised rib) is placed between the vertebral body and the anterior longitudinal ligament to span the diseased area (Fig. 7).

Posterior bony reconstruction (fusion) is performed at this stage. The remaining part of the rib is bivalved. The rib is placed posteriorly to bridge the laminectomy defect (if laminectomy is performed) or across the laminae of the diseased vertebrae. The posterior parts of the sublami- nar wires are bent over the graft to prevent it from being dislodged (Fig. 8).

\section{5) Closure}

A chest tube is inserted, and the chest is closed. The intact paravertebral (erector spinae) muscle provides a tight medial seal as well. Another drainage tube is inserted into the posteriorly exposed area (near the posterior midline); a standard posterior closure is performed. The medial reflected flap is then properly sutured to its lateral counterpart. The cue ends of the muscles are approximated properly using interrupted sutures. The subcutaneous tissue and skin are sutured.

\section{6) Postoperative management}

The patient is advised to change positions while in bed to prevent pressure necrosis of the wound. Drains are re-

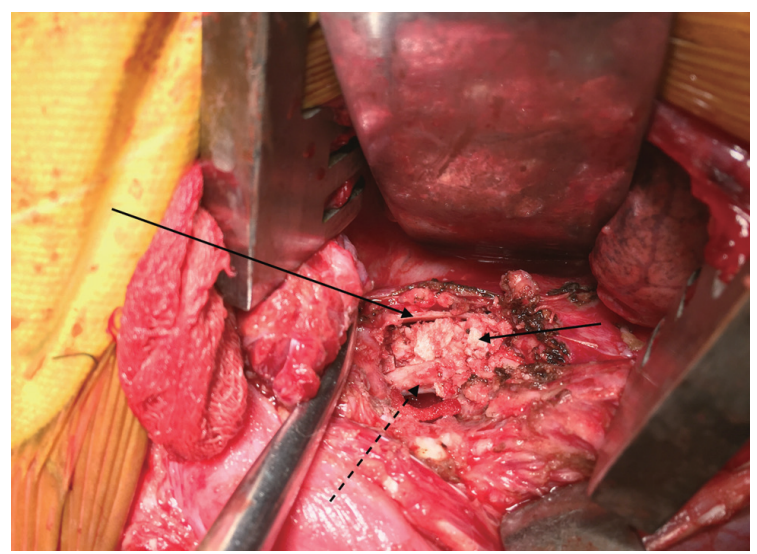

Fig. 7. Anterior reconstruction. A strut graft is placed between healthy proximal and distal bone (dashed arrow). An onlay graft is placed between the anterior longitudinal ligament and vertebral body (left arrow). Corticocancellous bone chips are placed between the onlay and strut grafts.

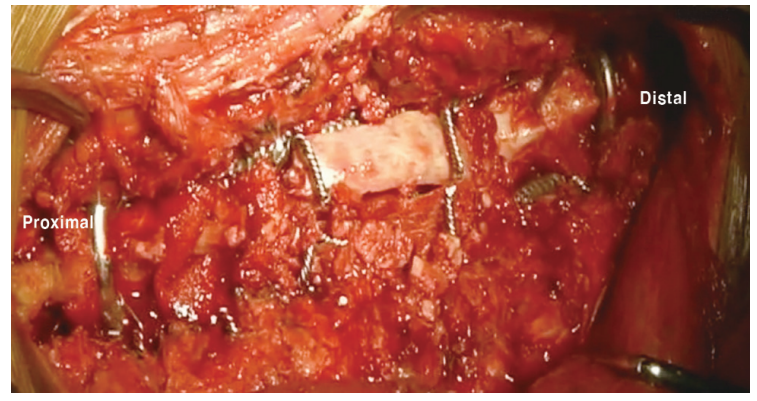

Fig. 8. Posterior reconstruction. A bivalve rib is placed posteriorly to bridge the laminectomy defect (if laminectomy was performed) or across the laminae of the diseased vertebrae. The posterior parts of the sublaminar wires are bent over the graft to prevent it from being dislodged. 
moved after output becomes minimal. Twenty-four hours prior to chest drain removal, the drain is clamped and an $\mathrm{X}$-ray is taken to rule out pleural fluid accumulation. The patient is mobilized after drain removal using a thoracolumbo-sacral orthosis (TLSO). Standing and walking are started once the patient regains ambulatory power.

The radiological outcome of one of the patients treated using this approach is shown in Fig. 1. Intraoperative samples from all patients were sent for culture and sensitivity. ATT was administered for 18 months. In cases of drug-resistant tuberculosis, ATT was administered for 2 years.

\section{Results}

All 143 patients underwent a minimum follow-up of 18 months, with a mean follow-up of $60.23 \pm 24.56$ months (range, 18-156 months). During presentation, the mean value of preoperative kyphosis was $24.02^{\circ} \pm 8.89^{\circ}$ (range, $9^{\circ}-45^{\circ}$ ). The average number of vertebral bodies involved was $2.36 \pm 0.57$ (range, 2-4). D5-6 was the most common vertebral level involved. The distribution of involved levels is shown in Table 1.

Estimated blood loss was $489 \pm 124 \mathrm{~mL}$ (range, $250-850$ $\mathrm{mL}$ ). Single-level laminectomy was required for $18 \mathrm{pa}-$ tients, and two-level laminectomy was required for 3 patients. No laminectomy was needed for the remaining 122 patients. The total levels fixed were $5.90 \pm 1.01$ (range, $4-9)$. Anterior reconstruction was performed using only rib grafts in 69 patients, tricortical iliac crest bone grafts plus ribs in 72 patients, and fibulas plus ribs in 2 pediatric patients.

The mean immediate postoperative $\mathrm{K}$ angle was $10.35^{\circ} \pm$ $4.96^{\circ}$ (range, $1^{\circ}-21^{\circ}$ ). Improvements in $\mathrm{K}$ angle were statistically significant $(p<0.001)$. At the latest follow-up, the mean $\mathrm{K}$ angle was $12.55^{\circ} \pm 5.07^{\circ}$ (range, $3^{\circ}-28^{\circ}$ ), with an average correction loss of $2.19^{\circ} \pm 2.11^{\circ}\left(\right.$ range, $\left.0^{\circ}-25^{\circ}\right)$. The average percentage of correction at the latest follow-up was $43.57 \% \pm 21.99 \%$ (range, $0 \%-91 \%$ ).

Twelve patients did not have any preoperative neurological deficits: Their neurological status was unchanged postoperatively. All eight patients with Frankel grade D recovered completely. Out of 85 patients with Frankel grade C, 77 patients recovered fully and 8 patients recovered to grade D. Out of 38 patients with Frankel grades A and B, 25 patients recovered completely, 12 patients recovered to grade $\mathrm{D}$, and 1 patient remained paraplegic. No patient
Table 1. Distribution of the level involved in tuberculosis of dorsal spine

\begin{tabular}{lrc}
\hline Levels involved & Frequency & Percent \\
\hline D1-3 & 7 & 4.9 \\
\hline D2-3 & 12 & 8.4 \\
\hline D2-4 & 4 & 2.8 \\
\hline D2-5 & 4 & 2.8 \\
\hline D3-4 & 7 & 4.9 \\
\hline D3-5 & 4 & 2.8 \\
\hline D3-6 & 3 & 2.1 \\
\hline D4-5 & 12 & 8.4 \\
\hline D4-6 & 3 & 2.1 \\
\hline D5-6 & 36 & 25.2 \\
\hline D5-7 & 7 & 4.9 \\
\hline D6-7 & 3 & 2.1 \\
\hline D6-8 & 12 & 8.4 \\
\hline D7-8 & 11 & 7.7 \\
\hline D8-10 & 4 & 2.8 \\
\hline D8-9 & 7 & 4.9 \\
\hline D9-10 & 6 & 4.2 \\
\hline D10-12 & 1 & 0.7 \\
\hline
\end{tabular}

Table 2. Preoperative and postoperative neurological status of patients

\begin{tabular}{llll}
$\begin{array}{l}\text { Preoperative Frankel } \\
\text { stage (no. of patients) }\end{array}$ & $\begin{array}{l}\text { Postoperative Frankel } \\
\text { stage (no. of patients) }\end{array}$ \\
$A(28)$ & $E(17)$ & $D(10)$ & $A(1)$ \\
$B(10)$ & $E(8)$ & $D(2)$ & \\
$C(85)$ & $E(77)$ & $D(8)$ \\
$D(8)$ & $E(8)$ & \\
$E(12)$ & $E(12)$ & \\
\hline
\end{tabular}

deteriorated following surgery. Patient neurological status, both pre- and postoperatively, is shown in Table 2.

Pain improved from an average VAS score of 7.02 \pm 0.97 (range, 5-9) preoperatively to $1.51 \pm 0.59$ (range, $1-3$ ) at the final follow-up. Improvements were statistically significant $(p<0.01)$. Fusion was achieved in all cases.

Eight patients had superficial maceration of skin wounds, which healed after daily cleaning and dressing. One patient developed buckling of the anterior rib graft, with kyphosis reappearance (Fig. 9). However, because this patient recovered neurologically from Frankel grade $\mathrm{D}$ to grade $\mathrm{A}$ and she had a negligible cosmetic deformity, revision surgery was not opted for. She was mobilized with a TLSO until a union was achieved. One patient presented 
with distal-most sublaminar wire breakage, following a traffic accident. An X-ray revealed local kyphosis reappearance (Fig. 10). He was managed by revision "hybrid fixation" (Fig. 10D). The broken sublaminar wire was removed; a pedicle screw was inserted at both that level and one level distal to it. The limbs of the Hartshill rectangle were inserted into the head of the pedicle screw, and the locking nuts were tightened. The patient made an uneventful recovery.
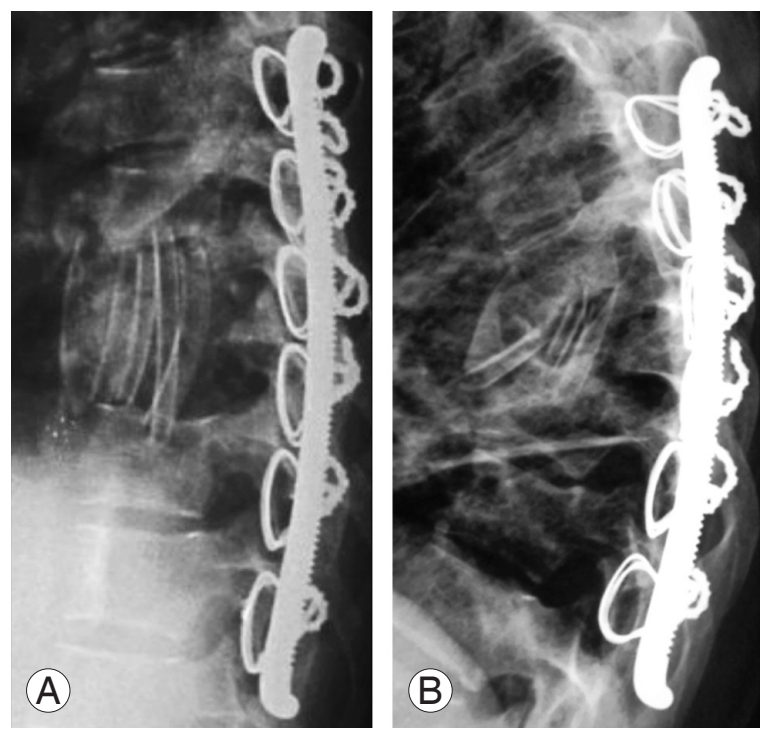

Fig. 9. Radiographs showing a complication. (A) Immediate postoperative X-ray showing anterior reconstruction performed with multiple rib grafts. (B) Buckling of the anterior rib graft and localized kyphosis reappearance.

\section{Discussion}

The most common form of tuberculosis of the spine is the paradiscal type with anterior column involvement. Various approaches have been described to treat tuberculosis of the spine, including anterior, posterior, and combined approaches. The anterior approach has the advantage of direct visualization of the anterior column for debridement, without disturbing the normal posterior elements. Anterior reconstruction is better achieved using the anterior approach. However, the posterior approach allows superior fixation than the anterior approach. Localized kyphosis correction is also improved if fixation is performed on the tensile (posterior surface) surface [12].

An all-posterior approach results in a biomechanically stronger fixation. However, for anterior necrotic tissue debridement and anterior reconstruction, some normal posterior elements (e.g., pedicle and transverse process) must be sacrificed. Because anterior debridement and reconstruction are performed via the posterolateral window, the advantage of the direct visualization of the reconstruction is not obtained using an all-posterior approach. The combined approach has the advantages of providing a biomechanically sound fixation and direct visualization of the anterior reconstruction, but it requires two separate incisions and a change in position of the patient. A combined anterior-posterior approach has a longer surgical time, greater blood loss, and longer hospitalization than
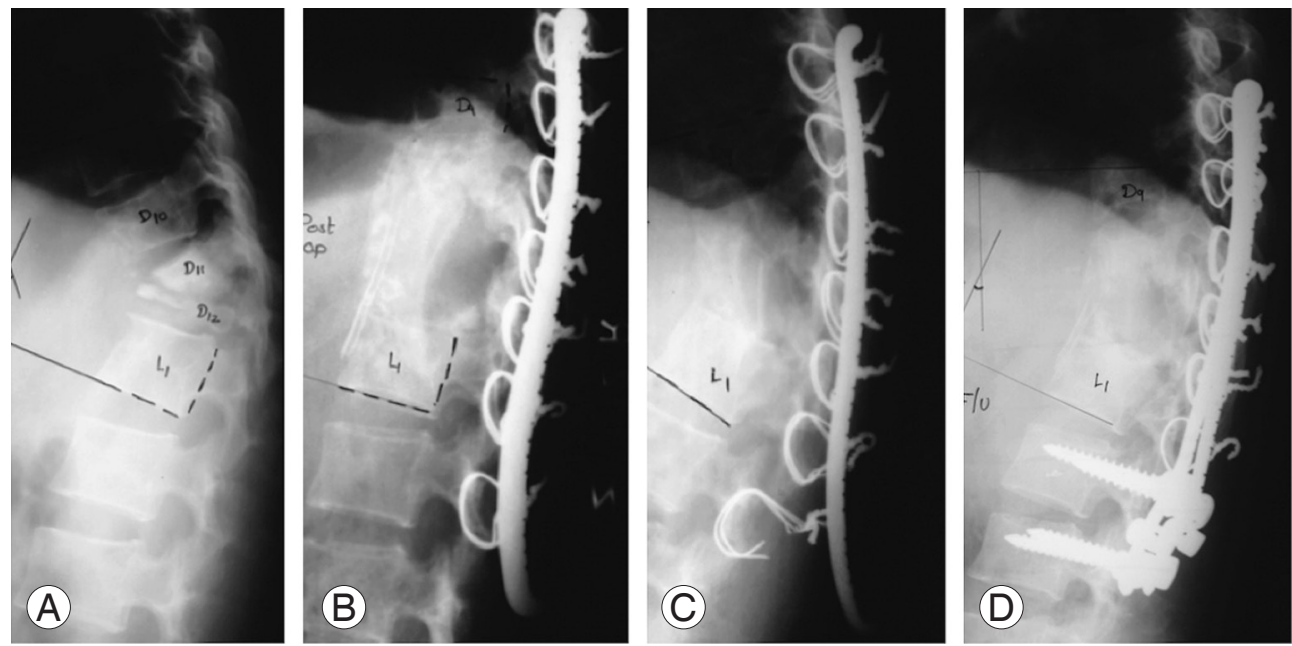

Fig. 10. Implant failure. (A) Preoperative X-ray (lateral view), showing complete D12 collapse and partial D11 collapse, with localized kyphosis. (B) Postoperative X-ray showing localized kyphosis correction and anterior reconstruction with a tricortical bone and onlay graft using an excised rib. Posterior fixation was performed using sublaminar wires and a Hartshill rectangle. (C) X-ray after road traffic accident, showing breakage of the distal sublaminar wire and local kyphosis reappearance. (D) Revision fixation performed by "Hybrid fixation." 
the posterior approach alone $[7,13]$.

The approach described in this study is a single incision using a combined anterior-posterior approach. Using a single incision and the same patient position, this method offers the advantages of anterior and posterior approaches. Direct visualization of the anterior debridement and reconstruction can be achieved without sacrificing the normal posterior elements. We believe that facet joints are some of the strongest stabilizing structures in the spine; therefore, saving the facet joints helps preserve the inherent spine stability. We believe that using the "Versatile approach", the normal facet joints are protected, thereby preserving some inherent spinal stability. Global spinal cord decompression and subsequent spinal reconstruction and fusion can be achieved because anterior and posterior columns are simultaneously exposed. Because fixation is performed on the posterior (tensile) surface, it is biomechanically stable. The disadvantage of this approach is that pedicular screw fixation is not recommended because the patient is placed in the lateral position. However, because we experienced only one implant failure, we feel that fixation with sublaminar wires and a Hartshill rectangle is adequate if done properly. Fixation with a Hartshill rectangle has the advantage of lower cost than with pedicular screws; hence, it is a suitable option, particularly in developing countries.

We would like to emphasize that sound anterior reconstruction is the key to successful surgical management of tuberculosis of the spine. The "Versatile approach" offers the advantage of direct visualization of the anterior reconstruction. After all anterior necrotic tissue removal and posterior fixation, the anterior void can be seen directly. Further debridement may be performed until healthy bone is visible on both ends. A slot is made in the proximal and distal bone prior to fitting the graft, ensuring that the graft snugly fits into the anterior column and providing a wide surface area for a successful union. However, as the bone is generally soft due to tuberculous abscesses, one must very gently make the slot using small, sharp instruments. If the void is small and the rib is not very soft or osteoporotic, anterior reconstruction may be performed via a rib graft only. If the anterior void is large and the rib quality is poor, a tricortical graft may be harvested from the iliac crest and used for anterior reconstruction. However, the use of only a single piece of rib would be inadequate to support the entire anterior column. Thus, if a rib graft is used, then as many pieces of rib as possible (minimum two pieces) should be used for the anterior reconstruction. If required, pieces of the rib may be tied together using nonabsorbable sutures. A mesh cage filled with corticocancellous bone may also be used. Additionally "onlay" grafting can be performed by inserting part of the excised rib between the vertebral body and anterior longitudinal ligament to increase the strength of the final union.

The "Versatile approach" allows for direct anterior reconstruction and permits global spinal cord decompression because anterior and posterior columns are fully exposed. Here laminectomy was performed in $21 / 143$ patients (15\%). We advocate PLL excision in patients with neurological deficits and no ambulatory power (Frankel grades A-C). PLL excision is easier by the "Versatile approach" than by an all-posterior approach, as PLL is directly visualized. Because anterior and posterior columns are exposed simultaneously, the lamina and all anterior necrotic tissue can be excised and this is termed as global decompression. Hence, the spinal cord will be adequately decompressed under direct visualization. The pedicle and transverse process need not be removed as these structures seldom cause cord compression. However, we advocate lamina removal only in cases where it is diseased and causes cord compression. If the lamina is normal, which is true for most cases of tuberculosis of the spine, anterior decompression alone will suffice. Of 131 patients with a neurological deficit, 130 patients (99.23\%) regained ambulatory power. Among these patients, 107 (82.30\%) recovered fully (Frankel E), whereas 23 patients $(17.70 \%)$ regained partial ambulatory power in their lower limbs (Frankel D). A good neurological outcome is likely the result of direct visualization of the anterior decompression of the spinal cord offered by the "Versatile approach". One patient with complete paraplegia did not improve. This patient had presented with a history of paraplegia since 12 months of age and had likely developed irreversible ischemia of the spinal cord.

A brief comparison of our patients is presented in Table 3. We have compared our results with those of other studies using anterior, posterior, and combined approaches [1422]. In recent years, the all-posterior approach has gained popularity. We would like to point out that neurological recovery after the posterior approach is inferior to the "Versatile approach". The percentage of patients who remained nonambulatory (Frankel grades A-C) after the posterior approach was $1.66 \%-18.9 \%$ (Table 3 ); in our series, only one patient $(0.69 \%)$ remained nonambulatory, even though our study is one of the largest case series (143 


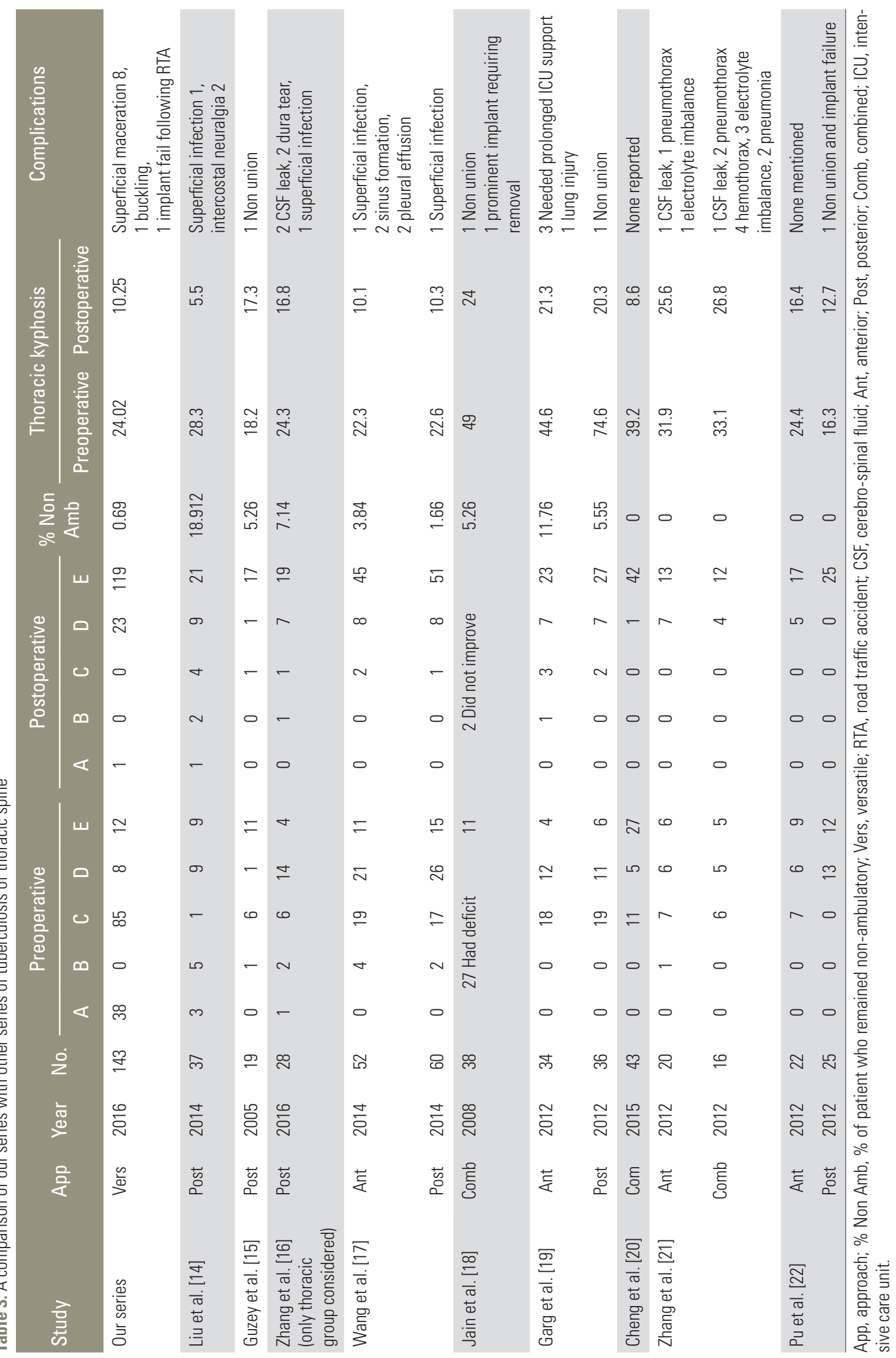


patients) on tuberculosis of the thoracic spine.

Additionally, we noted that 27/28 patients with Frankel grade A improved to Frankel grades D and E. This is a significant finding because such improvements in patients with Frankel grade A have seldom been reported. Because tuberculosis predominately occurs in the anterior column, we believe that adequate anterior decompression is important for neurological recovery, even in patients with a severe neurological deficit. Anterior debridement and decompression, with PLL excision, are easier to achieve using the "Versatile approach" than using an all-posterior approach because the anterior column can be visualized directly. This approach resulted in the good neurological outcomes observed in this series. However, it not possible to provide an unequivocal explanation for the significant findings of our study.

The advantages of the "Versatile approach" are as follows:

(1) Advantages of the anterior and posterior approaches are gained using a single stage, single incision, and single patient position.

(2) Direct visualization of the debridement and reconstruction of the anterior column is achieved.

(3) PLL is easy to excise when needed.

(4) Fixation can be applied to the tensile posterior surface, providing better biomechanical stability.

(5) A Hartshill rectangle (rectangle loop fixation) was used in all patients. As this implant is less expensive than pedicular screws, it meets a need at our institute, which caters to patients below the poverty line.

The disadvantage of the "Versatile approach" is that the lateral position is not a comfortable position for the patient during pedicular screw fixation. However, pedicle screw fixation in the lateral position has been described and may be attempted. Because we experienced only one implant failure, we feel that fixation with sublaminar wires and a Hartshill rectangle is adequate if done properly.

The limitation of our study is that it is a retrospective case series. Because of the absence of a control group, direct comparison of anterior and posterior approaches could not be done. Future prospective, comparative studies may provide additional insight into the advantages and disadvantages of these procedures.

\section{Conclusions}

The "Versatile approach" is an effective single-stage, single-incision, combined anterior-posterior approach for the surgical treatment of tuberculosis of the thoracic spine. It offers the advantage of direct visualization of the decompression and reconstruction of anterior and posterior vertebral columns, thereby providing excellent longlasting clinical outcomes.

\section{Conflict of Interest}

No potential conflict of interest relevant to this article was reported.

\section{References}

1. Fanning A. Tuberculosis: 6. Extrapulmonary disease. CMAJ 1999;160:1597-603.

2. Sharma SK, Mohan A. Extrapulmonary tuberculosis. Indian J Med Res 2004;120:316-53.

3. Moon MS. Tuberculosis of spine: current views in diagnosis and management. Asian Spine J 2014;8:97111.

4. Jain AK, Kumar J. Tuberculosis of spine: neurological deficit. Eur Spine J 2013;22 Suppl 4:624-33.

5. Hodgson AR, Stock FE. Anterior spine fusion for the treatment of tuberculosis of the spine: the operative findings and results of treatment in the first 100 cases. Bone Joint J Surg 1960;42:295-310.

6. Upadhyay SS, Saji MJ, Yau ACMC. Duration of antituberculosis chemotherapy in conjunction with radical surgery in the management of spinal tuberculosis. Spine 1996;21:1898-903.

7. Zhang HQ, Lin MZ, Shen KY, et al. Surgical management for multilevel noncontiguous thoracic spinal tuberculosis by single-stage posterior transforaminal thoracic debridement, limited decompression, interbody fusion, and posterior instrumentation (modified TTIF). Arch Orthop Trauma Surg 2012;132:751-7.

8. Louw JA. Spinal tuberculosis with neurological deficit: treatment with anterior vascularised rib grafts, posterior osteotomies and fusion. J Bone Joint Surg Br 1990;72:686-93.

9. Moon MS, Woo YK, Lee KS, Ha KY, Kim SS, Sun DH. Posterior instrumentation and anterior interbody fusion for tuberculous kyphosis of dorsal and lumbar spines. Spine (Phila Pa 1976) 1995;20:1910-6.

10. Tuli SM. Results of treatment of spinal tuberculosis by "middle-path" regime. J Bone Joint Surg Br 1975; 57:13-23. 
11. Jain AK, Dhammi IK. Tuberculosis of the spine: a review. Clin Orthop Relat Res 2007;460:39-49.

12. Jain AK, Jain S. Instrumented stabilization in spinal tuberculosis. Int Orthop 2012;36:285-92.

13. Zhang H, Zeng K, Yin X, Huang J, Tang M, Guo C. Debridement, internal fixation, and reconstruction using titanium mesh for the surgical treatment of thoracic and lumbar spinal tuberculosis via a posterior-only approach: a 4-year follow-up of 28 patients. J Orthop Surg Res 2015;10:150.

14. Liu Z, Liu J. One-stage posterior debridement and transpedicular screw fixation for treating monosegmental thoracic and lumbar spinal tuberculosis in adults. ScientificWorldJournal 2014;2014:137106.

15. Guzey FK, Emel E, Bas NS, et al. Thoracic and lumbar tuberculous spondylitis treated by posterior debridement, graft placement, and instrumentation: a retrospective analysis in 19 cases. J Neurosurg Spine 2005;3:450-8.

16. Zhang P, Peng W, Wang X, et al. Minimum 5-year follow-up outcomes for single-stage transpedicular debridement, posterior instrumentation and fusion in the management of thoracic and thoracolumbar spinal tuberculosis in adults. Br J Neurosurg 2016; 30:666-71.

17. Wang X, Pang X, Wu P, Luo C, Shen X. One-stage anterior debridement, bone grafting and posterior instrumentation vs. single posterior debridement, bone grafting, and instrumentation for the treatment of thoracic and lumbar spinal tuberculosis. Eur Spine J 2014;23:830-7.

18. Jain AK, Dhammi IK, Jain S, Kumar J. Simultaneously anterior decompression and posterior instrumentation by extrapleural retroperitoneal approach in thoracolumbar lesions. Indian J Orthop 2010;44:40916.

19. Garg B, Kandwal P, Nagaraja UB, Goswami A, Jayaswal A. Anterior versus posterior procedure for surgical treatment of thoracolumbar tuberculosis: a retrospective analysis. Indian J Orthop 2012;46:165-70.

20. Cheng Z, Wang J, Zheng Q, Wu Y, Guo X. Anterolateral radical debridement and interbody bone grafting combined with transpedicle fixation in the treatment of thoracolumbar spinal tuberculosis. Medicine (Baltimore) 2015;94:e721.

21. Zhang HQ, Li JS, Zhao SS, et al. Surgical management for thoracic spinal tuberculosis in the elderly: posterior only versus combined posterior and anterior approaches. Arch Orthop Trauma Surg 2012;132: 1717-23.

22. $\mathrm{Pu} \mathrm{X}, \mathrm{Zhou} \mathrm{Q}, \mathrm{He} \mathrm{Q}$, et al. A posterior versus anterior surgical approach in combination with debridement, interbody autografting and instrumentation for thoracic and lumbar tuberculosis. Int Orthop 2012; 36:307-13. 